\title{
Screening agars for MRSA: evaluation of a stepwise diagnostic approach with two different selective agars for the screening for methicillin-resistant Staphylococcus aureus (MRSA)
}

Volker Micheel ${ }^{1 \dagger}$, Benedikt Hogan ${ }^{2 \dagger}$, Thomas Köller ${ }^{3}$, Philipp Warnke ${ }^{3}$, Sabine Crusius ${ }^{3}$, Rebecca Hinz ${ }^{1}$, Ralf Matthias Hagen ${ }^{1}$, Norbert Georg Schwarz ${ }^{2}$ and Hagen Frickmann ${ }^{1,3^{*}}$

\begin{abstract}
Background: Colonization with methicillin-resistant Staphylococcus aureus (MRSA) poses a hygiene risk that does not spare field hospitals or military medical field camps during military deployments. Diagnostic options for unambiguously identifying MRSA isolates are usually scarce in military environments. In this study, we assessed the stepwise application of two different selective agars for the specific identification of MRSA in screening analyses.

Methods: Nasal swabs from 1541 volunteers were subjected to thioglycollate broth enrichment and subsequently screened on CHROMagar MRSA selective agar for the identification of MRSA. The MRSA identity of suspicious-looking colonies was confirmed afterwards or excluded by another selective agar, chromID MRSA. All isolates from the selective agars with MRSA-specific colony morphology were identified by biochemical methods and mass spectrometry.

Results: The initial CHROMagar MRSA screening identified suspicious colonies in 36 out of 1541 samples. A total of 25 of these 36 isolates showed MRSA-like growth on chromID agar. Out of these 25 isolates, 24 were confirmed as MRSA, while one isolate was identified as Staphylococcus kloosii. From the 11 strains that did not show suspicious growth on chromID agar, 3 were methicillin-sensitive Staphylococcus aureus (MSSA, with one instance of co-colonization with Corynebacterium spp.), 2 were confirmed as MRSA (with 1 instance of co-colonization with MSSA), 2 were lost during passaging and could not be re-cultured, one could not be identified by the applied approaches, and the remaining 3 strains were identified as Staphylococcus saprophyticus, Staphylococcus hominis (co-colonized with Macrococcus caseolyticus) and Staphylococcus cohnii, respectively.
\end{abstract}

Conclusions: The application of the selective agar CHROMagar MRSA alone proved to be too non-specific to allow for a reliable diagnosis of the presence of MRSA. The combined use of two selective agars in a stepwise approach reduced this non-specificity with an acceptably low loss of sensitivity. Accordingly, such a stepwise screening approach might be an option for resource-restricted military medical field camps.

Keywords: Methicillin-resistant Staphylococcus aureus, Selective agar, Chromogenic agar, Screening, Hygiene, Stepwise diagnostics, CHROMagar, ChromID agar

\footnotetext{
* Correspondence: Frickmann@bni-hamburg.de

${ }^{\dagger}$ Equal contributors

'Department of Tropical Medicine at the Bernhard Nocht Institute, German

Armed Forces Hospital of Hamburg, Hamburg, Germany

${ }^{3}$ Institute for Microbiology, Virology and Hygiene, University Medicine

Rostock, Rostock, Germany

Full list of author information is available at the end of the article
} 


\section{Background}

Colonization with methicillin-resistant Staphylococcus aureus (MRSA) poses a relevant public health threat worldwide. Soldiers are at risk as well, both during exercises and on deployment. MRSA is readily transmissible and shows high tenacity, potentially leading to outbreak situations as previously occurred for military trainees in San Diego, California [1]. The nostrils and mucous membranes of the upper respiratory tract are typical sites of colonization [2] from which nosocomial spread may occur. Such colonization with MRSA has been demonstrated for US soldiers irrespective of their current deployment situations [3].

The disease spectrum caused by Staphylococcus aureus ranges from mild to moderate skin and soft tissue infections to life-threatening systemic infections [4]. Impressively high percentages of MRSA-induced abscesses have been identified in American soldiers [5].

Increasing numbers of MRSA strains have been isolated in hospitals and community settings since the introduction of beta-lactam antibiotics [6, 7]. Once introduced, community-associated MRSA strains can readily spread within healthcare facilities [8] and pose a considerable risk for immunologically compromised trauma patients within military medial field hospitals or camps on deployment.

Reliable screening procedures are needed for the deployment setting to prevent the spread of MRSA from colonized patients by the enforcement of adequate hygiene precautions. Point-of-care PCR systems such as GeneXpert (Cepheid, Sunnyvale, California) deliver rapid results [9]. However, the specificity of the GeneXpert system has been shown to be reduced in cases of mecA gene loss $[10,11]$; furthermore, such point-of-care devices are not always available.

Chromogenic selective agars are inexpensive and ready-to-use alternatives or amendments [12] if the basic laboratory infrastructure needed for the growth of bacteria can be provided. Numerous evaluations of MRSAselective agars have been introduced [13-56] with acceptable but still improvable results regarding their sensitivities and specificities. In this study, we evaluated a stepwise approach using one selective agar as a screening method and another agar for confirmation testing.

\section{Methods}

A total of 1541 nasal swabs for MRSA screening (agar gel transport single plastic swabs without charcoal, Amies w/o Ch, Copan Italia SpA, Brescia, Italy, 108C.USE) were included in the analysis. The nasal vestibulum of healthy adult volunteers was screened using one swab per study participant. The study population comprised Madagascan civilians. To ensure the privacy of the volunteers, no further details regarding the sampling setting are presented for this technical evaluation in accord with our institutes' ethical standards, which prohibit the assessment and presentation of patient data that do not directly impact the scientific question being assessed. The descriptive analysis of the laboratory procedures presented here does not demand a more detailed presentation of the patient data.

After broth enrichment of the samples in thioglycollate broth (BD, Heidelberg, Germany) for $16-24 \mathrm{~h}$ at $37^{\circ} \mathrm{C}$, the cultures were grown on the MRSA selective agar CHROMagar MRSA (MAST Diagnostica, Reinfeld, Germany) for $40-48 \mathrm{~h}$ at $37^{\circ} \mathrm{C}$ as an initial screening step. Suspicious colonies on the CHROMagar MRSA plates, i.e., mauve-colored colonies, were selected and stored at $-80{ }^{\circ} \mathrm{C}$ in Microbank tubes (Pro-Lab Diagnostics, Bromborough, UK) until further assessment.

As a next step, all isolated strains were grown on another MRSA-selective chromogenic agar, chromID MRSA (bioMérieux, Marcy-l'Étoile, France, reference: Best.-Nr.43 451 (20 units) or Best.-Nr.43 459 (100 units)), for an additional $40-48 \mathrm{~h}$ at $37^{\circ} \mathrm{C}$ to confirm or contradict the result of the CHROMagar MRSA-based screening. The isolates were grown directly from the Microbank tubes.

All suspicious isolates that were confirmed by chromID MRSA testing, i.e., green colonies on the chromID MRSA agar, were subjected to matrix-assisted laserdesorption-ionization time-of-flight mass-spectrometry (MALDI-TOF-MS) by a Shimadzu/Kratos "AXIMA Assurance" MALDI-TOF mass spectrometer (SHIMADZU Germany Ltd., Duisburg, Germany) as described [57] for confirmation on the species level. Spectral fingerprints were analyzed using Vitek MS IVD V2 database MS-CE version CLI 2.0.0 (bioMérieux). Commercial BD MAX MRSA PCR (BD, Heidelberg, Germany) was used to verify the identity as MRSA.

Isolates from the CHROMagar MRSA that were not confirmed as MRSA by the growth of suspicious colonies on chromID MRSA were subjected to the following: repeated growth on chromID MRSA agar, penicillin-binding protein 2a (pbp-2a)-latex agglutination (PBP2a Culture Colony Test, Alere Scarborough Inc., Scarborough, Maine, USA), Pastorex Staph-Plus (Bio-Rad, Marnes-la-Coquette, France) latex agglutination targeting clumping factor, protein A and capsular polysaccharides, VITEK-II GP-card (bioMérieux)-based identification and MALDI-TOF-MS (as described above). Antimicrobial susceptibility testing of Staphylococcus aureus strains was performed using the VITEK-II AST-P619-card (bioMérieux) to confirm oxacillin resistance.

For MRSA strains that were incorrectly missed by the chromID MRSA agar confirmation testing, spa typing was performed using the Ridom StaphType standard protocol for PCR and subsequent double-strand sequencing $[58,59]$. Automated sequence allocation was performed 
using the software Ridom StaphType version 2.2.1 (Ridom Ltd., Würzburg, Germany).

This study was approved by the Ethical Committee of the Ministry of Health of the Republic of Madagascar prior to being conducted (No. $081-$ MSANP/CE, $5^{\text {th }}$ November 2012).

\section{Results and discussion}

From 36 out of the analyzed 1541 nasal swabs from healthy volunteers, MRSA-suspicious colonies were isolated from the CHROMagar plates after thioglycollate broth enrichment and freezing at $-80{ }^{\circ} \mathrm{C}$ in Microbank tubes. As reported from previous studies, the sensitivity and specificity of this selective agar for MRSA ranges from $95.4 \%$ to $100 \%$ and $95 \%$ to $100 \%$, respectively [60-62]. From these 36 strains, 2 did not grow after freezing, so their identity could not be assessed. It is thus theoretically possible that these strains were indeed MRSA.

For the remaining 34 strains, 25 grew on the chromID MRSA agar as MRSA-like green colonies. The previously described ranges of sensitivity and specificity of chromID MRSA agar are $64.5 \%$ to $99.4 \%$ and $98.5 \%$ to $99.4 \%$, respectively, for the detection of MRSA [63]. From the 25 strains showing green colonies, 24 out of 25 were confirmed as Staphylococcus aureus by MALDI-TOFMS and as MRSA by the commercial BD MAX MRSA PCR. However, one strain was misidentified by the combined approach based on CHROMagar MRSA and chromID MRSA agar. MALDI-TOF-MS analysis identified this strain as Staphylococcus kloosii, a coagulase-negative skin colonizer. For the remaining 9 samples, no growth of the culture or growth of non-suspicious white colonies were observed on chromID MRSA agar. Photographs showing the typical examples of the colony morphology on CHROMagar MRSA for 4 out of these 9 samples are depicted in Fig. 1.

These 9 samples were further investigated by mass spectrometry and biochemical approaches. As described in previous reports [61], small purple colonies of skincolonizing Corynebacterium spp. on CHROMagar MRSA medium might be misidentified as potential MRSA strains by non-experienced investigators.

For one sample, the identification of tiny colonies failed. For three samples, the colonies were identified as coagulase-negative staphylococci, while the VITEK-II GP-cards and MALDI-TOF-MS unanimously identified Staphylococcus saprophyticus, Staphylococcus hominis and Staphylococcus cohnii in these cases. From the sample containing the Staphylococcus hominis strain, the skin-colonizer Macrococcus caseolyticus was also isolated and identified by mass spectrometry. In another three samples, methicillin-sensitive Staphylococcus aureus (MSSA) was confirmed; methicillin-susceptibility was monitored by pbp-2a-agglutination and VITEK-II AST-619 antimicrobial susceptibility testing. Corynebacterium spp. was observed to be co-colonized with MSSA in one instance.

MRSA was confirmed in two of nine samples, in spite of an initial non-suspicious growth on chromID MRSA agar. However, after repeated growth on chromID MRSA agar, typical green colonies were identified. One of these two MRSA strains did not show characteristically green colonies earlier than $48 \mathrm{~h}$ of growth; an additional MSSA strain was isolated from this same sample. The reasons remain unclear why those two additional MRSA strains did not show typical colony color and morphology during the first assessment on chromID MRSA agar. A possible reason might be the fact that the first growth on chromID MRSA agar was performed directly from the Microbank tubes, as strains can tend to grow atypically immediately after thawing after prolonged storage at $-80{ }^{\circ} \mathrm{C}$.

To further characterize the two MRSA strains that were missed by initial chromID MRSA agar confirmation testing, spa typing $[58,59]$ was performed. This rapid and easy-to-use sequence-based molecular tool allows for international phylogenetic comparisons and has widely replaced pulsed field gel electrophoresis as the new gold standard of Staphylococcus aureus typing in Germany [64]. The identified spa types were t314 for the strain that grew after $48 \mathrm{~h}$ of incubation on chromID MRSA agar only and t186 for the other strain. The t314 clone is known to be prevalent in Western Africa [65] and the t186 clone in Eastern Africa [66].

In summary, MRSA strains were identified by the applied procedures in 26 of 1541 samples. A combined approach of CHROMagar MRSA selective agar as a screening tool and chromID MRSA agar as a confirmation tool after thioglycollate broth enrichment identified 24 out of 26 MRSA strains but initially missed two strains. In contrast, a Staphylococcus kloosii strain, which is rarely isolated from human samples, was erroneously considered to be MRSA.

However, one incorrectly attributed strain out of a total of 1541 samples represents an acceptable specificity of the approach. The total sensitivity cannot be estimated, as there was no comparative gold standard for the CHROMagar MRSA screening. Accordingly, one cannot say how many MRSA strains were missed by the initial screening.

The significant advantage of CHROMagar MRSA is the fact that the manufacturer sells the agar not only as ready-to-use agar plates but also as a powder, allowing for easy shipment without proper cooling and production of the plates at the site of deployment. However, if the storage of the powder for up to 2 years is intended, the manufacturer recommends storage at $2{ }^{\circ} \mathrm{C}$ to $8{ }^{\circ} \mathrm{C}$. The high number of false positive results due to 

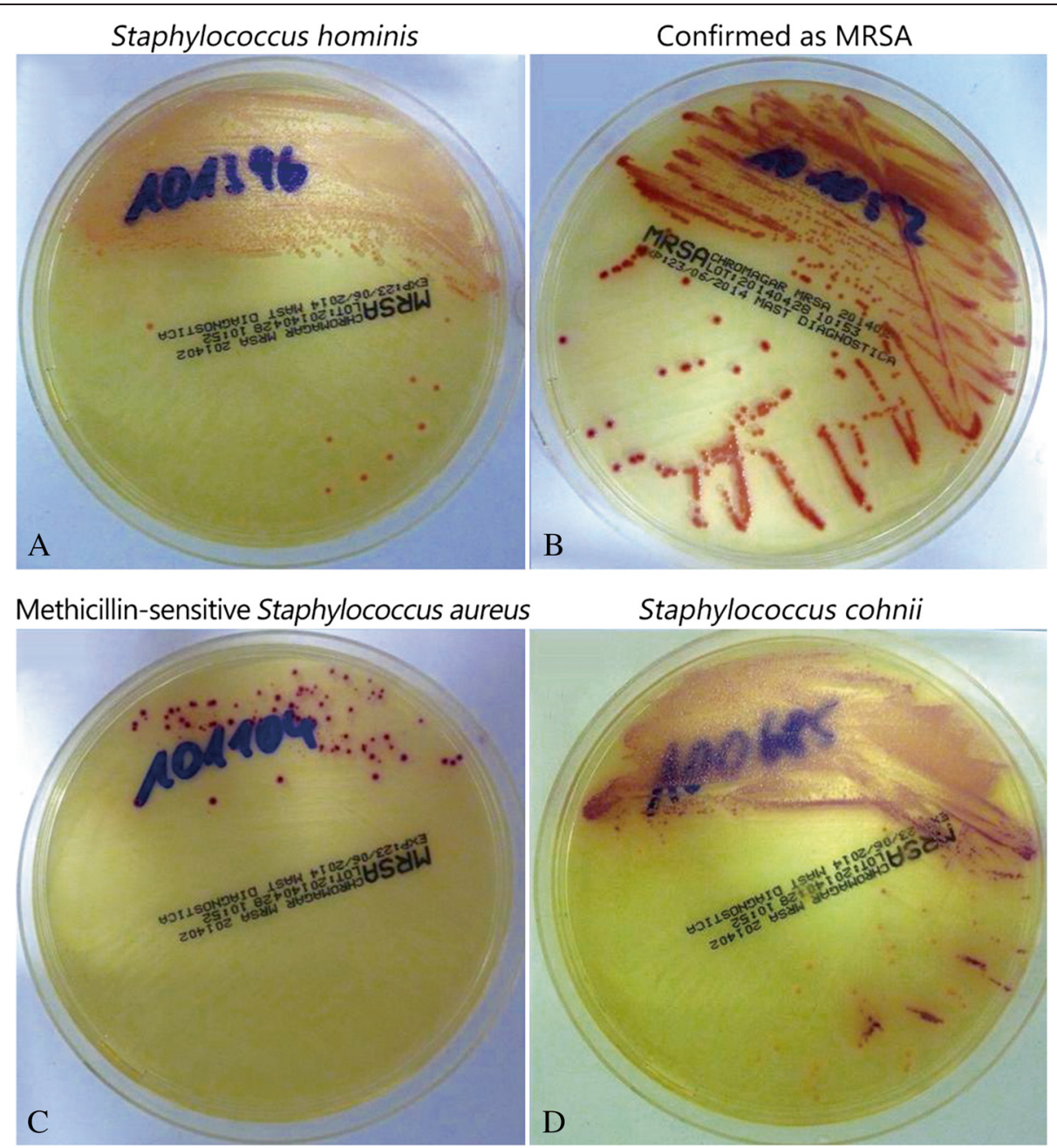

Fig. 1 Difficult to discriminate: colony morphology as observed during the CHROMagar MRSA screening. a) Staphylococcus hominis. b) Confirmed as MRSA. c) Methicillin-sensitive Staphylococcus aureus. d) Staphylococcus cohnii

CHROMagar MRSA screening alone is a point of concern, resulting in an insufficient specificity. The design of the study does not allow for statements on the specificity of a screening based on the solitary use of chromID MRSA agar, as this selective agar was used for confirmation testing only.

However, this was not the only limitation of this study. The fact that the initial inoculation was confined to just one MRSA screening agar was admittedly a major drawback. Accordingly, no proper comparison was possible. The stepwise approach described in this study was a description of field experience and not a comparative study.

The consequences of incorrect suspicions of colonization with MRSA are considerable. This is particularly true if isolation of MRSA-positive patients is enforced as a strategy to prevent nosocomial spread in the field hospital. As shown in several studies $[67,68]$, the isolation of colonized patients leads to reduced nursing quality with poorer outcomes as a result. Accordingly, the usefulness of screening procedures has been discussed with much controversy [69]. The repeated disinfecting washing of all patients without regard to colonization status would be an alternative regarding infection control [70-72] but has not yet been implemented widely.

\section{Conclusions}

If MRSA screening is needed in a resource-restricted medical field camp environment where no alternative approaches for reliable confirmation testing are available, screening with two different MRSA selective agars is an option with an acceptable specificity. CHROMagar MRSA powder can be easily shipped to sites of tropical deployment but bears the risk of misidentifying coagulasenegative staphylococci and MSSA as MRSA if it is used without confirmation testing. Due to the harmful consequences of isolation of MRSA-colonized patients, incorrect identification of colonization with MRSA should be avoided. 


\section{Abbreviations}

AST: Antibiotic susceptibility testing; MALDI-TOF-MS: Matrix-assisted laser desorption-ionization time-of-flight mass spectrometry; MRSA: Methicillin-resistant Staphylococcus aureus; MSSA: Methicillin-sensitive Staphylococcus aureus; PBP: Penicillin binding protein; PCR: Polymerase chain reaction; SPP: Species (plural).

\section{Competing interests}

The authors declare that there is no conflict of interest according to the guidelines of the International Committee of Medical Journal Editors.

\section{Authors' contributions}

VM participated in the laboratory experiments and the assessment of their results. $\mathrm{BH}$ collected the samples and participated in writing the manuscript. TK participated in the laboratory experiments and the assessment of their results. PW participated in the laboratory experiments and the assessment of their results as well as in writing the manuscript. SC participated in the laboratory experiments and the assessment of their results. RH participated in writing the manuscript. $\mathrm{RMH}$ was responsible for the study design and participated in writing the manuscript. NGS participated in the assessment of the study results. HF was responsible for the study design and participated in result assessment and writing of the manuscript. All authors have read and approved the final manuscript.

\section{Acknowledgements}

Annett Michel and Steffen Lohr are gratefully acknowledged for excellent technical assistance.

\section{Author details}

${ }^{1}$ Department of Tropical Medicine at the Bernhard Nocht Institute, German Armed Forces Hospital of Hamburg, Hamburg, Germany. ${ }^{2}$ Department of Infectious Disease Epidemiology, Bernhard Nocht Institute for Tropical Medicine Hamburg, Hamburg, Germany. Institute for Microbiology, Virology and Hygiene, University Medicine Rostock, Rostock, Germany.

Received: 9 December 2014 Accepted: 24 June 2015

Published online: 21 July 2015

\section{References}

1. Campbell KM, Vaughn AF, Russell KL, Smith B, Jimenez DL, Barrozo CP, et al. Risk factors for community-associated methicillin-resistant Staphylococcus aureus infections in an outbreak of disease among military trainees in San Diego, California, in 2002. J Clin Microbiol. 2004;42:4050-403.

2. Albrich WC, Harbarth S. Health-care workers: source, vector, or victim of MRSA? Lancet Inf Dis. 2008:8:289-301.

3. Vento TJ, Calvano TP, Cole DW, Mende K, Rini EA, Tully CC, et al. Staphylococcus aureus colonization of healthy military service members in the United States and Afghanistan. BMC Infect Dis. 2013;13:325.

4. Chambers HF, Deleo FR. Waves of resistance: Staphylococcus aureus in the antibiotic era. Nature Rev Microbiol. 2009;7:629-41.

5. Dufresne GW, Wells RD, Pfaff JA. The retrospective prevalence of community-acquired methicillin-resistant Staphylococcus aureus in soft tissue abscesses at two military level I trauma centers. Mil Med. 2008;173:945-8.

6. Mediavilla JR, Chen L, Mathema B, Kreiswirth BN. Global epidemiology of community-associated methicillin resistant Staphylococcus aureus (CA-MRSA). Curr Opin Microbiol. 2012;15:588-95.

7. Humphreys H, Grundmann H, Skov R, Lucet JC, Cauda R. Prevention and control of methicillin-resistant Staphylococcus aureus. Clin Microbiol Inf. 2009;15:120-4

8. Gonzalez BE, Rueda AM, Shelburne 3rd SA, Musher DM, Hamill RJ, Hulten KG. Community-associated strains of methicillin-resistant Staphylococccus aureus as the cause of healthcare-associated infection. Infect Control Hosp Epidemiol. 2006;27:1051-6.

9. Rossney AS, Herra CM, Brennan Gl, Morgan PM, O'Connell B. Evaluation of the Xpert methicillin-resistant Staphylococcus aureus (MRSA) assay using the GeneXpert real-time PCR platform for rapid detection of MRSA from screening specimens. J Clin Microbiol. 2008;46:3285-90.

10. Ciardo DE, Burger S, Payer M, Lee C, McCallum N. GeneXpert captures unstable methicillin-resistant Staphylococcus aureus prone to rapidly losing the mecA gene. J Clin Microbiol. 2010;48:3030-2.
11. Blanc DS, Basset P, Nahimana-Tessemo I, Jaton K, Greub G, Zanetti G. High proportion of wrongly identified methicillin-resistant Staphylococcus aureus carriers by use of a rapid commercial PCR assay due to presence of staphylococcal cassette chromosome element lacking the mecA gene. J Clin Microbiol. 2011;49:722-4.

12. Wassenberg MW, Kluytmans JA, Box AT, Bosboom RW, Buiting AG, van Elzakker EP, et al. Rapid screening of methicillin-resistant Staphylococcus aureus using PCR and chromogenic agar: a prospective study to evaluate costs and effects. Clin Microbiol Infect. 2010;16:1754-61.

13. Teramura H, Sekiguchi J, Shimojima M. Development of the novel chromogenic screening medium for methicillin-resistant Staphylococcus aureus. Diagn Microbiol Infect Dis. 2014;79:473-6.

14. Manickam K, Walkty A, Lagacé-Wiens PR, Adam H, Swan B, McAdam B, et al. Evaluation of MRSASelect $\left({ }^{\mathrm{T} M}\right)$ chromogenic medium for the early detection of methicillin-resistant Staphylococcus aureus from blood cultures. Can J Infect Dis Med Microbiol. 2013;24:e113-6.

15. Lee S, Park YJ, Park KG, Jekarl DW, Chae H, Yoo JK, et al. Comparative evaluation of three chromogenic media combined with broth enrichment and the real-time PCR-based Xpert MRSA assay for screening of methicillin-resistant Staphylococcus aureus in nasal swabs. Ann Lab Med. 2013:33:255-60

16. Pletinckx LJ, Dewulf J, De Bleecker Y, Rasschaert G, Goddeeris BM, De Man I. Evaluation of different chromogenic media for the detection of methicillin-resistant Staphylococcus aureus CC398 in broilers. Eur J Clin Microbiol Infect Dis. 2013;32:1023-6.

17. Veenemans J, Verhulst C, Punselie R, van Keulen PH, Kluytmans JA. Evaluation of brilliance MRSA 2 agar for detection of methicillin-resistant Staphylococcus aureus in clinical samples. J Clin Microbiol. 2013;51:1026-7.

18. Denys GA, Renzi PB, Koch KM, Wissel CM. Three-way comparison of BBL CHROMagar MRSA II, MRSASelect, and spectra MRSA for detection of methicillin-resistant Staphylococcus aureus isolates in nasal surveillance cultures. J Clin Microbiol. 2013;51:202-5.

19. Morris K, Wilson C, Wilcox MH. Evaluation of chromogenic meticillinresistant Staphylococcus aureus media: sensitivity versus turnaround time. J Hosp Infect. 2012;81:20-4.

20. Van Hoecke F, Deloof N, Claeys G. Performance evaluation of a modified chromogenic medium, ChromID MRSA New, for the detection of methicillin-resistant Staphylococcus aureus from clinical specimens. Eur J Clin Microbiol Infect Dis. 2011;30:1595-8.

21. Danial J, Noel M, Templeton KE, Cameron F, Mathewson F, Smith M, et al Real-time evaluation of an optimized real-time PCR assay versus Brilliance chromogenic MRSA agar for the detection of meticillinresistant Staphylococcus aureus from clinical specimens. J Med Microbiol. 2011;60(Pt 3):323-8

22. Buchan BW, Ledeboer NA. Identification of two borderline oxacillin-resistant strains of Staphylococcus aureus from routine nares swab specimens by one of three chromogenic agars evaluated for the detection of MRSA. Am J Clin Pathol. 2010;134:921-17.

23. Yang HY, Suh JT, Lee HJ. Evaluation of commercial selective agars in screening for methicillin-resistant Staphylococcus aureus. Ann Clin Lab Sci. 2010;40:252-6.

24. Cesur S, Yildiz E, Irmak H, Aygün Z, Karakoç E, Kinikli S, et al. Evaluation of oxacillin resistance screening agar and chromogenic MRSA agar media for the detection of methicillin resistance in Staphylococcus aureus clinical isolates. Mikrobiyol Bul. 2010;44:279-84 [Article in Turkish].

25. Peterson JF, Dionisio AA, Riebe KM, Hall GS, Wilson DA, Whittier S, et al. Alternative use for spectra MRSA chromogenic agar in detection of methicillin-resistant Staphylococcus aureus from positive blood cultures. J Clin Microbiol. 2010;48:2265-7.

26. Havill NL, Boyce JM. Evaluation of a new selective medium, BD BBL CHROMagar MRSA II, for detection of methicillin-resistant Staphylococcus aureus in stool specimens. J Clin Microbiol. 2010;48:2228-30.

27. Van Vaerenbergh K, Cartuyvels R, Coppens G, Frans J, Van den Abeele AM, De Beenhouwer $\mathrm{H}$, et al. Performance of a new chromogenic medium, BBL CHROMagar MRSA II (BD), for detection of methicillin-resistant Staphylococcus aureus in screening samples. J Clin Microbiol. 2010;48:1450-1.

28. Harrington AT, Mahlen SD, Clarridge 3rd JE. Significantly larger numbers of methicillin-resistant Staphylococcus aureus bacteria are recovered from polymicrobial respiratory and wound sites by use of chromogenic primary media than by use of conventional culture. J Clin Microbiol. 2010;48:1350-3 
29. Malhotra-Kumar S, Abrahantes JC, Sabiiti W, Lammens C, Vercauteren G, leven $\mathrm{M}$, et al. Evaluation of chromogenic media for detection of methicillin-resistant Staphylococcus aureus. J Clin Microbiol. 2010;48:1040-6.

30. Peterson JF, Riebe KM, Hall GS, Wilson D, Whittier S, Palavecino E, et al Spectra MRSA, a new chromogenic agar medium to screen for methicillinresistant Staphylococcus aureus. J Clin Microbiol. 2010;48:215-9.

31. Wolk DM, Marx JL, Dominguez L, Driscoll D, Schifman RB. Comparison of MRSASelect Agar, CHROMagar Methicillin-Resistant Staphylococcus aureus (MRSA) Medium, and Xpert MRSA PCR for detection of MRSA in Nares: diagnostic accuracy for surveillance samples with various bacterial densities. J Clin Microbiol. 2009;47:3933-6.

32. Bischof LJ, Lapsley L, Fontecchio K, Jacosalem D, Young C, Hankerd R, et al. Comparison of chromogenic media to BD GeneOhm methicillin-resistant Staphylococcus aureus (MRSA) PCR for detection of MRSA in nasal swabs. J Clin Microbiol. 2009;47:2281-3.

33. Carson J, Lui B, Rosmus L, Rennick H, Fuller J. Interpretation of MRSASelect screening agar at $24 \mathrm{~h}$ of incubation. J Clin Microbiol. 2009;47:566-8.

34. Tandé D, Garo B, Ansart S, Lejeune B. Efficiency of CHROMagar-MRSA in detecting meticillin-resistant Staphylococcus aureus in a routine setting. J Hosp Infect. 2008;70:388-9.

35. Nonhoff C, Denis O, Brenner A, Buidin P, Legros N, Thiroux C, et al. Comparison of three chromogenic media and enrichment broth media for the detection of methicillin-resistant Staphylococcus aureus from mucocutaneous screening specimens: Comparison of MRSA chromogenic media. Eur J Clin Microbiol Infect Dis. 2009;28:363-9.

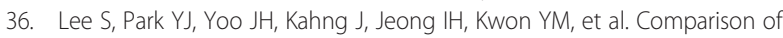
culture screening protocols for methicillin-resistant Staphylococcus aureus (MRSA) using a chromogenic agar (MRSA-Select). Ann Clin Lab Sci. 2008;38:254-7.

37. van Hal SJ, Jennings Z, Stark D, Marriott D, Harkness J. MRSA detection: comparison of two molecular methods (BD GeneOhm PCR assay and Easy-Plex) with two selective MRSA agars (MRSA-ID and Oxoid MRSA) for nasal swabs. Eur J Clin Microbiol Infect Dis. 2009;28:47-53.

38. Grandin S, Deschamps C, Magdoud F, Zihoune N, Branger C, Eveillard M. Evaluation of the impact of different lengths of pre-enrichment in a nutritive broth and prolonged incubation of MRSA-ID, a chromogenic agar medium, on its performances for identifying methicillin-resistant Staphylococcus aureus in screening samples. Pathol Biol (Paris). 2009;57:e37-42 [Article in French].

39. Krishna BV, Smith M, McIndeor A, Gibb AP, Dave J. Evaluation of chromogenic MRSA medium, MRSA select and Oxacillin Resistance Screening Agar for the detection of methicillin-resistant Staphylococcus aureus. J Clin Pathol. 2008;61:841-3.

40. Lagacé-Wiens PR, Alfa MJ, Manickam K, Harding GK. Reductions in workload and reporting time by use of methicillin-resistant Staphylococcus aureus screening with MRSASelect medium compared to mannitol-salt medium supplemented with oxacillin. J Clin Microbiol. 2008;46:1174-7.

41. Cherkaoui A, Renzi G, François P, Schrenzel J. Comparison of four chromogenic media for culture-based screening of meticillin-resistant Staphylococcus aureus. J Med Microbiol. 2007;56(Pt4):500-3.

42. van Loo $\mathrm{H}$, van Dijk S, Verbakel-Schelle I, Buiting AG. Evaluation of a chromogenic agar (MRSASelect) for the detection of meticillin-resistant Staphylococcus aureus with clinical samples in The Netherlands. J Med Microbiol. 2007:56(Pt4):491-4

43. Han Z, Lautenbach E, Fishman N, Nachamkin I. Evaluation of mannitol salt agar, CHROMagar Staph aureus and CHROMagar MRSA for detection of meticillin-resistant Staphylococcus aureus from nasal swab specimens. J Med Microbiol. 2007:56(Pt 1):43-6.

44. Compernolle V, Verschraegen G, Claeys G. Combined use of Pastorex Staph-Plus and either of two new chromogenic agars, MRSA ID and CHROMagar MRSA, for detection of methicillin-resistant Staphylococcus aureus. J Clin Microbiol. 2007:45:154-8.

45. Louie L, Soares D, Meaney H, Vearncombe M, Simor AE. Evaluation of a new chromogenic medium, MRSA select, for detection of methicillin-resistant Staphylococcus aureus. J Clin Microbiol. 2006:44:4561-3.

46. Harriau P, Ruffel F, Lardy JB. Use of BioRad plating agar MRSASelect for the daily detection of methicillin resistant staphylococci isolated from samples taken from blood culture bottles. Pathol Biol (Paris). 2006;54:506-9 [Article in French].

47. Diederen $B M$, van Leest CM, van Duijn I, Willemse $\mathrm{P}$, van Keulen $\mathrm{PH}$, Kluytmans JA. Evaluation of S. aureus ID, a chromogenic agar medium for the detection of Staphylococcus aureus. Infection. 2006;34:95-7.
48. Ben Nsira S, Dupuis M, Leclercq R. Evaluation of MRSA Select, a new chromogenic medium for the detection of nasal carriage of methicillinresistant Staphylococcus aureus. Int J Antimicrob Agents. 2006;27:561-4.

49. Nguyen Van JC, Kitzis MD, Ly A, Chalfine A, Carlet J, Ben Ali A, et al. Detection of nasal colonization methicillin-resistant Staphylococcus aureus: a prospective study comparing real-time genic amplification assay vs selective chromogenic media. Pathol Biol (Paris). 2006;54:285-92. Article in French].

50. Stoakes L, Reyes $R$, Daniel J, Lennox G, John MA, Lannigan $R$, et al. Prospective comparison of a new chromogenic medium, MRSASelect, to CHROMagar MRSA and mannitol-salt medium supplemented with oxacillin or cefoxitin for detection of methicillin-resistant Staphylococcus aureus. J Clin Microbiol. 2006:44:637-9.

51. Diederen BM, van Leest ML, van Duijn I, Willemse P, van Keulen PH, Kluytmans JA. Performance of MRSA ID, a new chromogenic medium for detection of methicillin-resistant Staphylococcus aureus. J Clin Microbiol. 2006;44:586-8

52. Flayhart D, Hindler JF, Bruckner DA, Hall G, Shrestha RK, Vogel SA, et al. Multicenter evaluation of BBL CHROMagar MRSA medium for direct detection of methicillin-resistant Staphylococcus aureus from surveillance cultures of the anterior nares. J Clin Microbiol. 2005;43:5536-40.

53. Perry JD, Davies A, Butterworth LA, Hopley AL, Nicholson A, Gould FK. Development and evaluation of a chromogenic agar medium for methicillin-resistant Staphylococcus aureus. J Clin Microbiol. 2004;42:4519-23.

54. Taguchi H, Kaneko T, Onozaki M, Kubo R, Kamiya S. Evaluation of a new chromogenic medium for isolation of MRSA. Kansenshogaku Zasshi. 2004;78:54-8. Article in Japanese].

55. Apfalter P, Assadian O, Kalczyk A, Lindenmann V, Makristathis A, Mustafa S, et al. Performance of a new chromogenic oxacillin resistance screen medium (Oxoid) in the detection and presumptive identification of methicillin-resistant Staphylococcus aureus. Diagn Microbiol Infect Dis. 2002;44:209-11.

56. Merlino J, Leroi M, Bradbury R, Veal D, Harbour C. New chromogenic identification and detection of Staphylococcus aureus and methicillinresistant S. aureus. J Clin Microbiol. 2000;38:2378-80.

57. Frickmann H, Christner M, Donat M, Berger A, Essig A, Podbielski A, et al. haemolyticus by fluorescence in situ hybridization (FISH) and two matrix-assisted laser-desorption-ionization time-of-flight mass spectrometry (MALDI-TOF-MS) platforms. PLoS One. 2013;8:e63222.

58. Harmsen D, Claus H, Witte W, Rothganger J, Claus H, Turnwald D, et al. Typing of methicillin-resistant Staphylococcus aureus in a university hospital setting by using novel software for spa repeat determination and database management. J Clin Microbiol. 2003;41:5442-8.

59. Mellmann A, Weniger T, Berssenbrügge C, Rothgänger J, Sammeth M, Stoye J, et al. Based Upon Repeat Pattern (BURP): an algorithm to characterize the long-term evolution of Staphylococcus aureus populations based on spa polymorphisms. BMC Microbiol. 2007;7:98.

60. Diederen B, van Duijn I, van Belkum A, Willemse P, van Keulen P, Kluytmans J. Performance of CHROMagar MRSA Medium for detection of Methicillinresistant Staphylococcus aureus. J Clin Microbiol. 2005;43:1925-7.

61. Loulergue J, de Gialluly C, Morange V, Hostein A, van der Mee-Marquet N, Quentin R. Evaluation of a new chromogenic medium for isolation and presumptive identification of Methicillin-resistant Staphylococcus aureus from human clinical specimens. Eur J Clin Microbiol Inf Dis. 2006:25:407-9.

62. Rahbar M, Islami P, Saremi M. Evaluation of a new CHROMagar medium for detection of Methicillin-resistant Staphylococcus aureus. PJBS. 2008;11:496-8.

63. Verkade E, Ferket M, Kluytmans J. Clinical evaluation of Oxoid Brilliance MRSA agar in comparison with bioMerieux MRSA ID medium for detection of livestock-associated Methicillin-resistant Staphylococcus aureus. J Med Microbiol. 2011;60(Pt 7):905-8.

64. Frickmann H, Gawlik PP, Crusius S, Podbielski A. The current role of pulsedfield gel electrophoresis in Methicillin-resistant Staphylococcus aureus (MRSA) typing and the retrospective identification of outbreaks. Eu J Microbiol Imunol (Bp). 2012;2:128-33.

65. Egyr B, Guardabassi L, Sorum M, Nielsen SS, Kolekang A, Frimpong E, et al. Molecular epidemiology and antimicrobial susceptibility of clinical Staphylococcus aureus from healthcare institutions in Ghana. PLoS One. 2014;9:e89716.

66. Moremi N, Mshana SE, Kamugisha E, Kataraihya J, Tappe D, Vogel E, et al. Predominance of Methicillin resistant Staphylococcus aureus -St88 and new ST1797 causing wound infection and abscesses. J Infect Dev Ctries. 2012;6:620-5. 
67. Zahar JR, Garrouste-Orgeas M, Vesin A, Schwebel C, Bonadona A, Philippart $F$, et al. Impact of contact isolation for multidrug-resistant organisms on the occurrence of medical errors and adverse events. Intensive Care Med. 2013;39:2153-60

68. Morgan DJ, Diekema DJ, Sepkowitz K, Perencevich EN. Adverse outcomes associated with contact precautions: a review of the literature. Am J Infect Control. 2009;37:85-93.

69. Peterson $L R$, Diekema DJ. To screen or not to screen for methicillin-resistant Staphylococcus aureus. J Clin Microbiol. 2010;48:683-9.

70. Climo MW, Yokoe DS, Warren DK, Perl TM, Bolon M, Herwaldt LA, et al. Effect of daily chlorhexidine bathing on hospital-acquired infection. NEJM. 2013;368:533-42.

71. Huang SS, Septimus E, Kleinman K, Moody J, Hickok J, Avery TR, et al. Targeted versus universal decolonization to prevent ICU infection. NEJM. 2013;368:2255-65.

72. Derde LPG, Cooper BS, Goossens H, Malhotra-Kumar S, Willems RJ, Gniadkowsk $\mathrm{M}$, et al. Interventions to reduce colonisation and transmission of antimicrobialresistant bacteria in intensive care units: an interrupted time series study and cluster randomized trial. Lancet Inf Dis. 2014;14:31-9.

\section{Submit your next manuscript to BioMed Central and take full advantage of:}

- Convenient online submission

- Thorough peer review

- No space constraints or color figure charges

- Immediate publication on acceptance

- Inclusion in PubMed, CAS, Scopus and Google Scholar

- Research which is freely available for redistribution 